\title{
High Mortality in Hospitalized Elderly Patients with Feeding Tube Placement
}

\author{
Yasuharu TOKUDA and Hideki KoKETSU*
}

\begin{abstract}
Objective We investigated the influence of feeding tube placement on survival in hospitalized elderly patients.

Methods To assess long-term mortality in an inception cohort and the influence of feeding tube placement on survival, one hundred six hospitalized elderly patients from a nursing home were followed up through and after the index hospitalization for placement of a feeding tube and mortality. Cox regression hazards model was constructed for both univariate and multivariate analyses.

Results A feeding tube was placed in 15\% (16/106) of the study patients during the index hospitalization. Median survival of the 106 patients was 381 days. A total of 92 patients $(87 \%)$ survived the index hospitalization, and 52 $\mathbf{4 9 \% )}$ were still alive at the last follow-up. In the multivariate survival model which included older age, hip fracture history, admitting diagnosis of pneumonia, and tube feeding placement, only feeding tube placement (hazard ratio, 2.29 ; $95 \%$ confidence interval, $1.22-4.33$ ) was significantly associated with higher mortality.

Conclusion In hospitalized elderly patients from nursing home, feeding tube placement may be a risk factor for mortality.
\end{abstract}

(Internal Medicine 41: 613-616, 2002)

Key words: artificial nutrition, nursing home, prognosis

\section{Introduction}

Although recent studies and reviews of tube feeding in the care of elderly patients during hospitalization with acute illness (1) and with advanced dementia (2-5) found no clear and scientific basis for clinical effectiveness, placement of a feeding tube is a common practice (6-9). Decision making process must weigh the benefits and risks of tube feeding placement in this clinical setting. Precise knowledge of the probable out- comes of placing a feeding tube is also crucial for informed decision on behalf of patients and surrogates.

To this end, we analyzed long-term survival in an inception elderly cohort hospitalized from nursing home and cared for acute illnesses, and the influence of tube feeding on survival in this group of patients.

\section{Subjects and Methods}

Elderly patients aged 65 years or older admitted to Okinawa Chubu Hospital (a 550-bed community teaching hospital) from a nursing home in Okinawa, Japan during a 9-year period (March 1991-June 1999) were studied. Eligibility criteria included age of 65 years or older; hospitalization for an acute illness; residence in nursing home before hospitalization; and no previous history of feeding tube placement.

Informed consent was obtained from the designated surrogate decision maker (ie, health care agent or next of kin). The Institutional Review Board of Okinawa Chubu Hospital approved the study.

Initial assessment consisted of a complete medical history and physical examination by an attending physician. We gathered medical record information on demographics; preadmission medical history; admitting diagnosis; comorbidities, and functional status. Main outcome measures included mortality both during and after the index hospitalization, length of hospital stay, and feeding tube placement during the index hospitalization. These outcome measures were determined from the date of admission during the index hospitalization until discharge or in-hospital death. Follow-up telephone contact was maintained through June 1999 with next of kin of study subjects until subject's death was reported.

Statistical analysis focused on variables that predicted survival. To determine factors that influenced survival, we performed a series of single-variable Cox proportional hazards regression models examining the relationship of the variable of interest to time of death. Those variables included age, gender, comorbidities (dementia, hip fracture, and pressure ulcer), admitting diagnosis, and presence of a feeding tube. The rea-

From Division of General Internal Medicine, Department of Medicine, Okinawa Chubu Hospital, Okinawa *Department of Dermatology, Miyazaki Medical College, Miyazaki

Received for publication December 19, 2001; Accepted for publication April 12, 2002

Reprint requests should be addressed to Dr. Yasuharu Tokuda, Division of General Internal Medicine, Department of Medicine, Okinawa Chubu Hospital, 2083 Miyasato, Gushikawa, Okinawa 904-2293 
son we selected these variables for constructing a survival model was that they were suggested to be important prognostic factors for institutionalized elderly patients in a nursing home study (10). Variables of borderline significance $(\mathrm{p}<0.20)$ and the presence of a feeding tube were entered into the final multivariate survival model. All of the $\mathrm{p}$ values presented were two-sided and statistical significance was defined as $\mathrm{p}<0.05$.

\section{Results}

One hundred six subjects were eligible to participate during the 9-year (March 1991-June 1999) study period. Characteristics of study subjects are given in Table 1 . The average age of the subjects was 84.9 years old (age range, 65-101 years), 61 (57.5\%) were women. The most common admitting diagnosis was pneumonia [ 59 subjects], followed by urinary tract infection [24], biliary tract infection [4] and soft tissue infection [3]. Activities of daily living in most patients were relatively poor and they were dependent on care-givers since they are all from nursing home as eligibility criteria for this study. Although body mass index was not determined because most patients were chair-bound or bed-ridden and could not stand up for height measurement, they were in general relatively undernourished as is typical nursing home elderly.

Of the 106 subjects, $16(15.1 \%)$ had a feeding tube placed during the index hospitalization and left with it. Since mostly resident physicians determined indications of tube placement for individual patients and there were no complete documen-

Table 1. Characteristics of 106 Elderly Hospitalized Patients

\begin{tabular}{lcc}
\hline & No. & $\%$ \\
\hline Median age (range), years & $85(65-101)$ & \\
Female & 61 & 57.5 \\
Dementia before admission to the hospital & 66 & 62.3 \\
Hip fracture before admission to the hospital & 21 & 19.8 \\
Pneumonia as admitting diagnosis & 59 & 55.7 \\
Death during index hospitalization & 14 & 13.2 \\
Feeding tube placement & 16 & 15.1 \\
Median length of stay in hospital (range), days & $12.5(1-197)$ & \\
Pressure ulcer during index hospitalization & 14 & 13.2 \\
\hline
\end{tabular}

tations for these indications in medical records, no clear standard criteria was identified. However, usual clinical practice at this hospital in terms of tube feeding placement for elderly patients suggested poor oral intake or frequent episodes of aspiration as the common indications.

Of the 106 subjects, ninety-two subjects (86.8\%) survived the index hospitalization and were discharged. Median survival of the 106 enrolled subjects was 381 days (range, 1-2,411 days). Fifty-two (49.1\%) were still alive at the time of the last followup contact (July 1, 1999). The hazard model is given in Table 2. Age of 90 years old and older (hazard ratio, 1.99; 95\% CI, 1.15-3.45) and placement of new feeding tube (hazard ratio, 2.57 ; 95\% CI, 1.39-4.79) were associated with mortality in the univariate model. However, only feeding tube placement was significantly associated with mortality in the multivariate model (hazard ratio, 2.29; 95\% CI, 1.22-4.33). The KaplanMeier cumulative survival curve of subjects with and without feeding tube is shown in Fig. 1. The curve clearly shows that the survival of the tube-fed group of patients was worse compared to the non-tube-fed group.

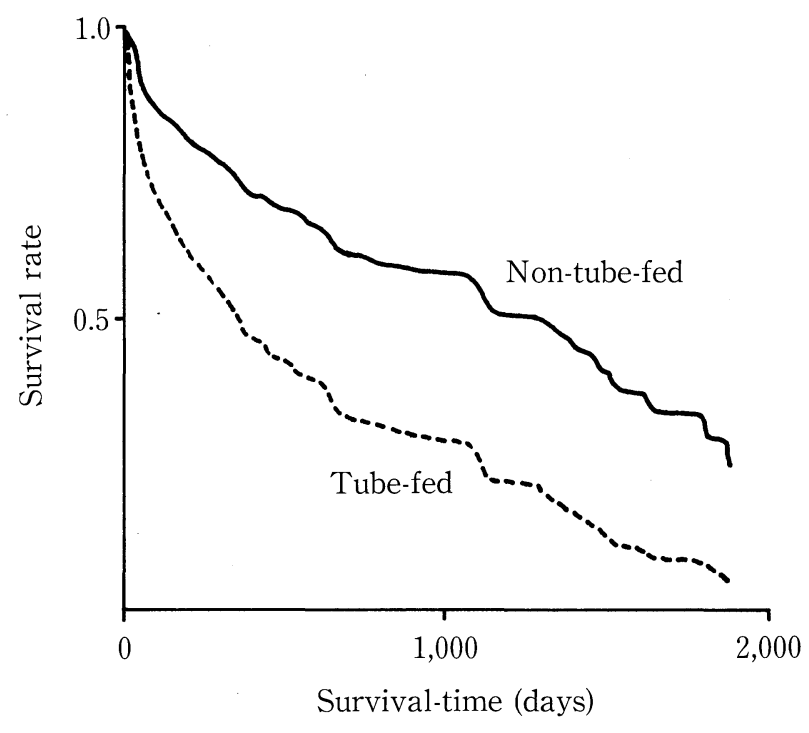

Figure 1. Kaplan-Meier survival comparing the tube-fed and non-tube-fed groups.

Table 2. Cox Proportional Hazards Regression Model for Mortality after Index Hospitalization in 106 Elderly Patients

\begin{tabular}{lccccccc}
\hline & \multicolumn{3}{c}{ Univariate model } & & & \multicolumn{3}{c}{ Multivariate model } \\
\cline { 2 - 3 } \cline { 6 - 8 } Risk factors & Hazard ratio & $95 \% \mathrm{CI}$ & $\mathrm{p}$ & & Hazard ratio & $95 \% \mathrm{CI}$ & $\mathrm{p}$ \\
\hline 90 years old and older & 1.99 & $1.15-3.45$ & 0.014 & & 1.75 & $0.99-3.09$ & 0.053 \\
Hip fracture before admission to the hospital & 1.5 & $0.80-2.81$ & 0.2 & & 1.38 & $0.73-2.63$ & 0.325 \\
Pneumonia as admitting diagnosis & 1.47 & $0.85-2.53$ & 0.164 & & 1.41 & $0.81-2.44$ & 0.220 \\
Tube feeding placement & 2.57 & $1.39-4.79$ & 0.003 & & 2.29 & $1.22-4.33$ & 0.010 \\
\hline
\end{tabular}

95\% CI: $95 \%$ confidence interval. 
Table 3. Clinical Characteristics of 16 Elderly Patients with Tube Feeding

\begin{tabular}{lccccc}
\hline Patient & Gender & Age $(\mathrm{yr})$ & Diagnosis & Outcome at last followup & Days from admission to last followup \\
\hline Patient 1 & F & 87 & pneumonia & dead & 2 \\
Patient 2 & F & 90 & pneumonia & dead & 40 \\
Patient 3 & M & 85 & pneumonia & dead & 70 \\
Patient 4 & F & 92 & UTI & dead & 138 \\
Patient 5 & F & 84 & pneumonia & dead & 155 \\
Patient 6 & F & 94 & cellulitis & dead & 177 \\
Patient 7 & F & 77 & UTI & dead & 232 \\
Patient 8 & M & 77 & UTI & dead & 278 \\
Patient 9 & F & 72 & cellulitis & dead & 366 \\
Patient 10 & F & 86 & BTI & alive & 383 \\
Patient 11 & M & 90 & UTI & dead & 537 \\
Patient 12 & M & 66 & pneumonia & dead & 757 \\
Patient 13 & M & 76 & pneumonia & dead & 891 \\
Patient 14 & M & 96 & UTI & dead & 1,102 \\
Patient 15 & F & 94 & pneumonia & alive & 1,340 \\
Patient 16 & M & 70 & pneumonia & dead & 1,391 \\
\hline
\end{tabular}

UTI : urinary tract infection, BTI : biliary tract infection.

Median survival during the index hospitalization and follow-up of subjects receiving a feeding tube was 322 days (range, 2-1,391 days) as compared with 420 days among subjects who did not receive a feeding tube (range, 1-2,411 days). Clinical characteristics of 16 elderly subjects with a tube feeding are shown in Table 3. Nine patients were women and median age was 85 (range 66-96 years of age). Leading admission diagnosis was pneumonia (8 patients). Since patient follow-up was conducted based on telephone contact with the family or surrogates after hospital discharge, the detailed causes related to death were not determined in this study.

\section{Discussion}

Recommendations about tube feeding should be based on its effectiveness. In our elderly hospitalized cohort from the nursing home, there was increased mortality among subjects who received a feeding tube during the index hospitalization as compared with those discharged from the hospital without a feeding tube.

The decision to use a feeding tube when oral intake cannot be easily maintained may be influenced by the intention of family and physicians to prevent the anticipated harms of malnutrition and dehydration (11). Inadequate intake of food and water is often thought to lead to hunger, thirst, and early death. Furthermore, tube feeding is believed to prevent aspiration pneumonitis and other infections, improve functional status, promote quality of care, and prolong life $(12,13)$.

However, recent reviews $(2,3,14)$ do not support these assumptions. Recent observational studies have confirmed high short-term mortality rates with tube feeding in the cohort of hospitalized patients in acute illness (1) and patients with advanced dementia $(2-5,14-17)$. Further, refusal of food and water in the patients of terminal illness is shown to be not usually harmful (18). The present study suggests that tube feeding actually has an adverse effect on long-term survival, at least in this cohort of elderly patients hospitalized with acute illness from nursing home.

It is now clear from multiple observational studies that feeding tubes do not prevent aspiration in patients with dementia (14). The continued risk of aspiration appears to result from reflux of gastric contents and aspiration of saliva and oropharyngeal secretion. Moreover, the hazardous consequence of feeding tube placement is the need to restrain the patient. The use of restraints is distressing to patients, even to persons with severe dementia, and it often results in agitation, which in turn may lead to the use of sedative medications with a great potential for adverse effects.

The limitations of this study include bias in the sample related to its conduct in a community teaching hospital in Okinawa, Japan. The standard practice for decisions to institute artificial nutrition and hydration under local tradition could lead to a different prevalence of feeding tube placement among patients compared with Western countries. However, elderly nursing home patients are commonly transferred to acute care hospitals for the treatment of acute illness (19) and the high risk of feeding tube placement and mortality is consistent with data from a wide range of geographic settings.

The present study suggested higher age, hip fracture history, and admission diagnosis of pneumonia as predictors of mortality only in the univariate model but not in multivariate model. The relatively small sample size may have limited our ability to identify additional predictors of mortality in this cohort.

We drew the following conclusions from this study. First, among a cohort of elderly hospitalized patients with acute ill- 
ness from nursing home, the risk of mortality is increased in patients receiving a new feeding tube in long-term follow-up. Second, there is a need to reach consensus on the indications for tube feeding in elderly patients presenting from nursing home setting to an acute care hospital. Third, the data presented here may facilitate the start of randomized controlled trials of feeding tube placement for elderly hospitalized patients with nutritional problems. Lastly, establishing evidence-based criteria for indications for tube feeding in the elderly nursing home patients admitted to an acute care hospital may reduce mortality and improve survival.

Acknowledgements: We thank Suresh K Joishy, MD for critical review of the manuscript.

\section{References}

1) Abuksis G, Mor M, Segal N, et al. Percutaneous endoscopic gastrostomy: high mortality rates in hospitalized patients. Am J Gastroenterol 95: 128$132,2000$.

2) Finucane TE, Christmas $C$, Travis $K$. Tube feeding in patients with advanced dementia: a review of the evidence. JAMA 282: 1365-1370, 1999.

3) Gillick MR. Rethinking the role of tube feeding in patients with advanced dementia. N Engl J Med 342: 206-210, 2000.

4) Meier DE, Ahronheim JC, Morris J, Baskin-Lyons S, Morrison RS. High short-term mortality in hospitalized patients with advanced dementia: lack of benefit of tube feeding. Arch Intern Med 161: 594-599, 2001.

5) Sanders DS, Carter MJ, D’Silva J, James G, Bolton RP, Bardhan KD. Survival analysis in percutaneous endoscopic gastrostomy feeding: a worse outcome in patients with dementia. Am J Gastroenterol 95: 1472-1475, 2000.

6) Callahan CM, Haag KM, Buchanan NN, Nisi R. Decision-making for percutaneous endoscopic gastrostomy among older adults in a community setting. J Am Geriatr Soc 47: 1105-1109, 1999.
7) Grant MD, Rudberg MA, Brody JA. Gastrostomy placement and mortality among hospitalized Medicare beneficiaries. JAMA 279: 1973-1976, 1998.

8) Rabeneck L, Wray NP, Petersen NJ. Long-term outcomes of patients receiving percutaneous endoscopic gastrostomy tubes. J Gen Intern Med 11: 287-293, 1996.

9) Ahronheim JC, Morrison RS, Baskin SA, Morris J, Meier DE. Treatment of the dying in the acute care hospital. Advanced dementia and metastatic cancer. Arch Intern Med 156: 2094-2100, 1996.

10) Walter LC, Brand RJ, Counsell SR, et al. Development and validation of a prognostic index for 1-year mortality in older adults after hospitalization. JAMA 285: 2987-2994, 2001.

11) Nair $\mathrm{S}$, Hertan H, Pitchumoni CS. Hypoalbuminemia is a poor predictor of survival after percutaneous endoscopic gastrostomy in elderly patients with dementia. Am J Gastroenterol 95: 133-136, 2000.

12) Bussone M, Lalo M, Piette F, Hirsch JF, Senecal P. Percutaneous endoscopic gastrostomy: its value in assisted alimentation in malnutrition in elderly patients. Apropos of 101 consecutive cases in patients over 70 years of age. Ann Chir 46: 59-66, 1992.

13) Kaw M, Sekas G. Long-term follow-up of consequences of percutaneous endoscopic gastrostomy (PEG) tubes in nursing home patients. Dig Dis Sci 39: 738-743, 1994.

14) Finucane TE, Bynum JP. Use of tube feeding to prevent aspiration pneumonia. Lancet 348: 1421-1424, 1996.

15) Mitchell SL, Kiely DK, Lipsitz LA. The risk factors and impact on survival of feeding tube placement in nursing home residents with severe cognitive impairment. Arch Intern Med 157: 327-332, 1997.

16) Mitchell SL, Kiely DK, Lipsitz LA. Does artificial enteral nutrition prolong the survival of institutionalized elders with chewing and swallowing problems? J Gerontol A Biol Sci Med Sci 53: M207-213, 1998.

17) Chouinard J, Lavigne E, Villeneuve $C$. Weight loss, dysphagia, and outcome in advanced dementia. Dysphagia 13: 151-155, 1998.

18) McCann RM, Hall WJ, Groth-Juncker A. Comfort care for terminally ill patients. The appropriate use of nutrition and hydration. JAMA 272: 12631266, 1994.

19) Fried TR, Mor V. Frailty and hospitalization of long-term stay nursing home residents. J Am Geriatr Soc 45: 265-269, 1997. 